\title{
Understanding starbursts through giant molecular clouds in high density environments
}

\author{
Erik W. Rosolowsky \\ Harvard-Smithsonian Center for Astrophysics \\ 60 Garden St., MS-66, Cambridge, MA 02138, USA \\ email:erosolow[snail]cfa.harvard.edu
}

\begin{abstract}
Starburst galaxies are characterized by uncommonly high star formation efficiencies, but it remains unclear what physical conditions in the molecular gas produce this high efficiency. Invariably, high star formation efficiency is associated with high column densities of molecular material (e.g. the Kennicutt-Schmidt law), but what are the conditions in the molecular clouds in starburst galaxies? Direct observations of starburst are difficult or impossible with current instruments, so I present the properties of GMCs in the Local Group as a starting case and then extend the analysis of GMC properties to nearby systems with surface densities of gas intermediate between the Local Group and starbursts. Rather than being constant, molecular cloud properties follow a continuum with significant variation across the Local Group and the intermediate surface density systems. Concomitant with these variations in the macroscopic properties are significant changes in the internal pressure and densities of molecular clouds, which implies significant variability in the initial conditions of the star formation process.
\end{abstract}

Keywords. galaxies: evolution, ISM: clouds, stars: formation

\section{Introduction}

While many phenomena may actively trigger star formation, the common feature of all these physical processes is the collection of gas into a small volume. On galactic scales, the "triggering" of star formation is best represented by the the Kennicutt-Schmidt law (Kennicutt 1998) which states that $\Sigma_{\mathrm{SFR}} \propto \Sigma_{\text {gas }}^{1.4}$ where $\Sigma_{\text {gas }}$ is the total surface density of gas. Subsequent studies have demonstrated that a more accurate relationship relates the surface density of star formation to the molecular gas: $\Sigma_{\text {SFR }} \propto \Sigma_{\text {mol }}^{1.4}$ (Murgia et al., 2002; Wong \& Blitz, 2002; Heyer et al., 2004). This star formation law spans over seven orders of magnitude in star formation rate stretching from $10^{-4}$ to $10^{3} M_{\odot} \mathrm{yr}^{-1} \mathrm{kpc}^{-2}$ with relatively little scatter $(<1 \mathrm{dex})$; for reference, the star formation rate in the solar neighborhood is $\sim 10^{-3} M_{\odot} \mathrm{yr}^{-1} \mathrm{kpc}^{-2}$ (Kroupa 1995). For systems with large-scale star formation rates comparable to that of the solar neighborhood, the star formation process likely follows the local pattern with stars forming in dense clumps of gas distributed through molecular clouds. However, for starburst systems where the star formation rate and gas surface density exceeds the solar neighborhood values by orders of magnitudes, star formation likely proceeds along significantly different lines, a conjecture borne out by the ubiquity of super star clusters in starburst systems (e.g. M82; O'Connell et al. 1995), which are never found in environments with low star formation rates. In addition, in starburst galaxies, the nature of the ISM must be significantly different. The neutral medium is almost entirely molecular in these systems; and the surface densities are sufficiently high $\left(>10^{2} M_{\odot} \mathrm{pc}^{-2}\right)$ that if GMCs were like those found locally, they would merge into a single continuous medium. Since the molecular gas in starbursts is the initial 
Table 1. Summary of GMC Properties in the Local Group and Transition Starburst Galaxies

\begin{tabular}{c|ccc} 
Galaxy & $\begin{array}{c}\sigma_{0} \\
{\left[\mathrm{~km} \mathrm{~s}^{-1}\right]}\end{array}$ & $\begin{array}{c}X_{\mathrm{CO}} \\
{\left[10^{20} \mathrm{~cm}^{-2} /\left(\mathrm{K} \mathrm{km} \mathrm{s}^{-1}\right)\right]}\end{array}$ & $\begin{array}{c}\Sigma_{\mathrm{H} 2} \\
{\left[M_{\odot} \mathrm{pc}^{-2}\right]}\end{array}$ \\
\hline LMC & 0.39 & 2.7 & 45 \\
SMC & 0.36 & 6.6 & 30 \\
M33 & 0.61 & 2.0 & 170 \\
IC10 & 0.55 & 1.7 & 140 \\
M31 & 0.72 & 2.6 & 200 \\
Outer MW & 0.40 & 3.0 & 50 \\
\hline M64 & 1.2 & 2.0 & 300 \\
M82 & $>1.9$ & $\lesssim 2.0$ & $\lesssim 500$ \\
MW Center & 2.6 & $<2.0$ & $<600$ \\
\hline Typical Errors & 0.5 & 0.05 & 10
\end{tabular}

conditions of their peculiar star and cluster formation, characterizing the properties of this gas is central to understanding the mechanics of the Kennicutt-Schmidt law. In these proceedings, I present some basic tools for characterizing the molecular ISM via scaling laws for giant molecular clouds (GMCs), present a summary of GMC properties in the Local Group galaxies for context, and then examine the GMCs in borderline starburst systems.

\section{Using Larson's laws to characterize GMC properties}

Larson (1981) first pointed out power law scalings between the macroscopic properties of molecular gas in a variety of systems. There are two independent "laws" expressed in a myriad of algebraically equivalent forms. In this paper, I will focus on the size-line width relationship and the virial parameter $(\alpha)$ :

$$
\sigma_{v}=\sigma_{0}\left(\frac{R}{1 \mathrm{pc}}\right)^{\beta}, \quad \alpha=\frac{5 R \sigma_{v}^{2}}{G M}
$$

where $\sigma_{v}$ is the one-dimensional velocity dispersion, $R$ is the cloud radius, and $M$ is the cloud mass. For GMCs in the solar neighborhood, $\sigma_{0} \approx 0.6 \mathrm{~km} \mathrm{~s}^{-1}, \beta=0.5$ and $\alpha \approx 1.5$ (Blitz 1993 and references therein). While the physical interpretation of these relationships continues to be debated, they remain a helpful parameterization of the similarities and differences among GMCs in a variety of environments. Recently, there has been a major effort to re-examine these relationships in Local Group galaxies (Blitz et al. 2005) while referencing all their measurements to a common standard, facilitating comparison among systems (Rosolowsky \& Leroy 2006). In Table 1, we summarize the size-line width relationship for GMCs in several galaxies across the Local Group. Since there are only a few points in each galaxy and a fit to the relationship is unstable, I only report the constant of proportionality $\left(\sigma_{0}\right)$ assuming the scaling indices are the same across systems. I also report the derived CO-to- $\mathrm{H}_{2}$ conversion factor $\left(X_{\mathrm{CO}}\right)$ that would be obtained if the virial parameter were $\alpha=1.5$ for all clouds as well as the mean surface density of the GMCs $\left(\Sigma_{\mathrm{H} 2}\right)$. There is significant variation in the GMC properties throughout the Local Group, as has been seen in previous studies (e.g., Rubio et al. 1993) and predicted based on environmental effects (Elmegreen 1989). These variations in the macroscopic properties of clouds imply substantial changes in the average internal properties of clouds such as their density and pressure - the average pressure in GMC in M31 is nearly 40 times that of a GMC in the SMC. 


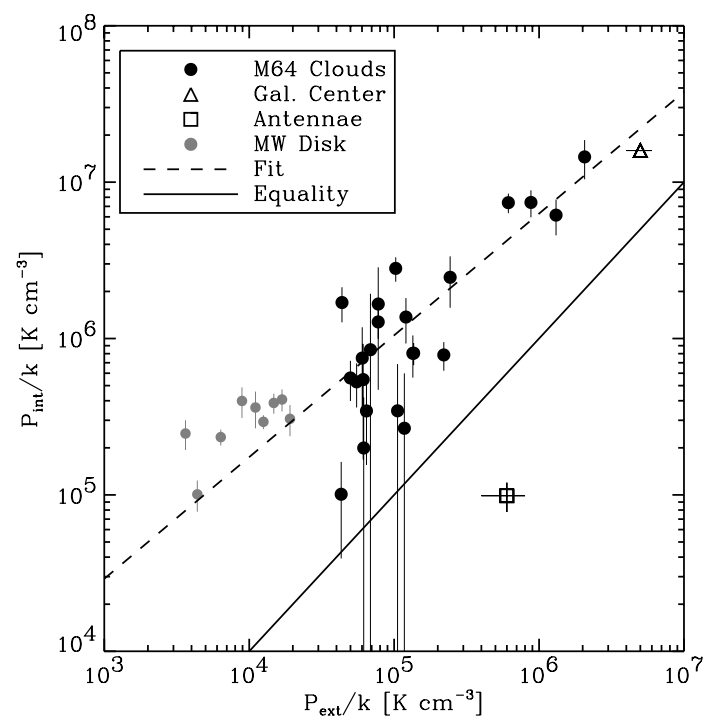

Figure 1. A comparison of internal and external pressures for GMCs in a variety of systems. Points represent pressure estimates in clouds for M64 (Rosolowsky \& Blitz 2005), Milky Way (Solomon et al. 1987), the Antennae galaxy (Wilson et al. 2004), and the Galactic center (Oka et al. 2001). Internal pressures are estimated from cloud properties. External pressures are quoted values from X-ray studies (Antennae, Galactic center) or estimated from the mass contributions of galactic components (Milky Way, M64). For most clouds, the internal pressure scales with the external pressure and is consistently above the derived value.

\section{GMCs in high density environments}

Ideally, we would compare the Local Group GMCs directly to clouds identified in starburst systems. Unfortunately, we cannot directly observe starbursts with the observational facilities currently available, owing to their limited angular resolution or insufficient sensitivity to map optically thin tracers. To make progress we must examine nearby systems that are closer (and therefore better resolved) and have surface densities intermediate between the disks of Local Group galaxies and starbursts. Three candidate systems are available for detailed study: the center of the Milky Way, M82 and M64. In Table 1, I present summary properties for these galaxies based off the analyses of Oka et al. (2001) for the galactic center, my own analysis of the data of Shen \& Lo (1995) and Keto et al. (2005) for M82, and Rosolowsky \& Blitz (2005) for M64. In these systems, the internal velocity dispersions are larger and the surface densities are higher implying an increased internal pressure of the GMCs. The internal pressure is consistently above estimates of the pressure in the ambient ISM and GMCs have internal pressures roughly an order of magnitude above that of the ambient ISM (Figure 1). In the high surface density systems, significantly more massive clouds are found: M64 harbors several GMCs with masses in excess of $10^{7} M_{\odot}$ which are unknown in the Local Group. Massive GMCs are necessary to support the formation of massive clusters $\left(>10^{6} M_{\odot}\right)$ with a reasonable efficiency.

For this limited number of systems, the molecular medium in the (transition) starburst systems supports significantly more massive clouds that are denser and more turbulent than the star-forming clouds seen in the Local Group. This general trend is merely the extension of variations in cloud properties seen throughout the Local Group. With the the parameterization of observational data becoming standardized across galaxies, future 
work should compare these variations in cloud properties to predictions of star formation based on theoretical models.

\section{Acknowledgements}

I am grateful to the National Science Foundation for their generous support of my work through an NSF Astronomy \& Astrophysics Postdoctoral Fellowship (AST-0502605).

\section{References}

Blitz, L. 1993, in: E.H. Levy \& J.I. Lunine (eds.), Protostars and Planets III (Tucson: Univ. Arizona), p. 125

Blitz, L., Fukui, Y., Kawamura, A., Leroy, A., Mizuno, N. \& Rosolowsky, E. 2006, in: B. Reipurth, D. Jewitt \& K. Keil (eds.), Protostars $\&$ Planets $V$, in press

Elmegreen, B. G. 1989, ApJ 338, 178

Heyer, M. H., Corbelli, E., Schneider, S. E. \& Young, J. S. 2004, ApJ 602, 723

Kennicutt, R. C. 1998, ApJ 498, 541

Keto, E., Ho, L. C. \& Lo, K.-Y. 2005, ApJ 635, 1062

Kroupa, P. 1995, MNRAS 277, 1522

Larson, R. B. 1981, MNRAS 194, 809

Murgia, M., Crapsi, A., Moscadelli, L. \& Gregorini, L. 2002, A\&SA 385, 412

O'Connell, R. W., Gallagher, III, J. S., Hunter, D. A. \& Colley, W. N. 1995, ApJ 446, L1

Oka, T., Hasegawa, T., Sato, F., Tsuboi, M., Miyazaki, A. \& Sugimoto, M. 2001, ApJ 562, 348

Rosolowsky, E. \& Blitz, L. 2005, ApJ 623, 826

Rosolowsky, E. \& Leroy, A. 2006, PASP 118, 590

Rubio, M., Lequeux, J. \& Boulanger, F. 1993, A\&A 271, 9

Shen, J. \& Lo, K. Y. 1995, ApJ 445, L99

Solomon, P. M., Rivolo, A. R., Barrett, J. \& Yahil, A. 1987, ApJ 319, 730

Wilson, C. D., Scoville, N., Madden \& S. C.,Charmandaris, V. 2003, ApJ 599, 1049

Wong, T. \& Blitz, L. 2002, ApJ 569, 157

\section{Discussion}

DIB: For the GMCs in the different galaxies is there also a difference in the exponent of the size-line width relationship?

Rosolowsky: We cannot distinguish a change in index from a change in the constant of proportionality with the quality of the data we have. However, we can say that at least one of the index or the constant varies among galaxies and perhaps both.

DiB: It would also be interesting to check the relationship between $\sigma_{0}$ and the star formation rate.

Rosolowsky: Particularly in dwarf galaxies where significant variation in both are observed.

Elmegreen: As long as you are looking at virialized clouds, this coefficient depends on pressure as a power law and pressure depends on ambient column density and therefore the star formation rate.

KENNICUTT: In fact, the metal-poor galaxies do have star formation rates that lie systematically above the mean Kennicutt(1998) Schmidt law. The offset is in the direction one would expect if the standard CO-to- $\mathrm{H}_{2}$ conversion factor underestimated the molecular gas density. So I believe that is consistent with the results presented in this talk. 